\section{Estudo dos efeitos auditivos e extraauditivos da exposição ocupacional a ruído e vibração}

\author{
Márcia Fernandes, Thaís Catalani Morata
}

1

Resumo / Summary

\section{Auditory and extra-auditory effects of occupational exposure to noise and vibration}

Palavras-chave: vibração, ruído,

perdas auditivas, prevenção.

Key words: vibration, noise, hearing loss, prevention. bjetivo: Este estudo teve como objetivo investigar as queixas de saúde e achados audiológicos de dois grupos de trabalhadores. Forma de estudo: Clínico prospectivo randomizado. Material emétodo: Grupo 1- exposto a níveis de pressão sonora elevados e vibração transmitida por meio das mãos-braços nos trabalhadores que operam motorroçadeiras, e Grupo 2- exposto a níveis elevados de pressão sonora e vibração transmitida por meio do corpo inteiro nos trabalhadores que operam equipamentos pesados como pácarregadeira, moto niveladora, retro-escavadeira e rolo compressor. Os 73 participantes passaram por entrevista, inspeção do meato acústico externo e audiometria tonal. Resultado: Em relação aos problemas de saúde, o grupo 2, exposto a vibração de corpo inteiro, é o que apresenta um maior número de queixas. Os trabalhadores do Grupo 1 , sem exceção, fazem uso do protetor auditivo e apenas 4 (11\%) referem zumbido. No Grupo 2 nem todos os trabalhadores usam o protetor auditivo e 6 (17\%) referem apresentar zumbido. Entretanto, a porcentagem de audiogramas alterados é mais elevada no grupo 1, expostos a vibração transmitida por meio das mãos-braços. Conclusão: Este estudo revelou uma série de deficiências no acompanhamento de saúde dessas populações e demonstrou a necessidade da implantação de programas preventivos tanto no que se refere à exposição a níveis de pressão sonora elevados quanto à vibração.

\begin{abstract}
A . The present study aimed to investigate the health complaints and the audiological findings of 2 groups of workers. Study design: Clinical prospective randomized. Material and method: Group 1 was exposed to high sound pressure levels and vibration transmitted by hands and arms through the use of power brush cutter/string trimmers. Group 2 was exposed to high sound pressure levels and wholebody vibration transmitted by heavy machinery such as vibrating compactor rollers, skid-steer loaders, backhoes and compact hydraulic excavators. The 73 participants underwent an interview, otoscopy, and pure-tone audiometry. Regarding general health, group 2 workers, exposed to whole-body vibration presented the highest number of complaints. Results: All the participants from group 1 use hearing protectors and $11 \%$ of them complained about tinnitus. Not all workers from group 2 use hearing protectors and $17 \%$ of them 2 reported tinnitus. However, group 1 workers, exposed to hand-arm vibration was the group with the highest percentage of abnormal audiograms. Conclusion: This study revealed a series of weaknesses in the health surveillance of these populations and indicated the need for the implementation of preventive programs focusing on their exposures to noise and vibration.
\end{abstract}




\section{INTRODUÇÃO}

Agentes físicos como ruído, calor, vibrações, pressões e radiações e agentes químicos como fumo, poeira, gases, vapores são alguns dos estressores ambientais encontrados em vários locais de trabalho. Os estressores organizacionais são fatores relacionados à organização do trabalho como, por exemplo, turnos, ritmo e ergonomia, ou seja, a relação do trabalhador com suas tarefas. Eles alteram o funcionamento de todo o organismo e o sono, aumentam a sensibilidade aos agentes estressores ambientais e, consequentemente, aumentam o risco de acidentes de trabalho. Combinados, esses estressores podem ter uma série de efeitos sobre a saúde e bem-estar dos trabalhadores.

Entre os agentes de risco ocupacionais destaca-se o ruído. Sabe-se que os trabalhadores expostos a este risco queixam-se de perda auditiva e zumbido, e de vários outros sintomas como cefaléia, nervosismo, problemas de estômago. A vibração também é um agente de risco ocupacional que, por sua complexidade e dificuldade na identificação da mesma e de seus efeitos sobre o homem, ainda merece ser estudada.

O objetivo do presente estudo foi o de analisar as queixas relatadas no que se refere à exposição ocupacional a vibrações e níveis elevados de pressão sonora, bem como os achados da avaliação audiológica, correlacionando ambos e analisando os aspectos relevantes para a prevenção das perdas auditivas.

\section{Perda auditiva induzida por ruído - PAIR}

Entende-se por perda auditiva induzida por ruído (PAIR) as alterações dos limiares auditivos do tipo neurossensorial, decorrentes da exposição ocupacional sistemática a níveis de pressão sonora elevados. Esta tem como características principais a irreversibilidade e a progressão gradual com o tempo de exposição ao risco. A sua história natural mostra, inicialmente, o acometimento dos limiares auditivos em uma ou mais freqüências da faixa de 3.000 a $6.000 \mathrm{~Hz}$. As demais freqüências poderão levar mais tempo para ser afetadas. Uma vez cessada a exposição, não haverá progressão da redução auditiva (Brasil, 1998).

Hétu e Phaneuf (1990) afirmam que, entre todas as deficiências auditivas, a PAIR é a patologia prevenível mais comum. Mas o ruído não é a única causa de perdas auditivas no ambiente de trabalho. Outros fatores também podem influenciar sua ocorrência. Entre eles, são citados: vibrações, exposição a agentes ototóxicos e temperaturas extremas (Morata e Lemasters, 1995). A exposição concomitante ao ruído, produtos químicos (Morata, 1986; Souza, 1994) e vibrações (Carnicelli, 1994), podem agravar a perda auditiva induzida por níveis de pressão sonora elevados. Hétu e Phaneuf (1990) relatam ainda que, quando foram formuladas recomendações para os níveis permitidos de exposição ao ruído, as perdas auditivas observadas foram atribuídas exclusivamente ao ruído, e outros agentes físicos e químicos foram e ainda têm sido ignorados. Os autores sugerem que mais estudos sejam realizados relacionando o ruído e outras exposições ocupacionais nocivas e deixam alguns questionamentos. Entre eles: "Os níveis permitidos de exposição ao ruído deveriam ser reduzidos na presença de outro agente causador da perda auditiva?"

\section{Vibração}

O corpo humano está exposto a vibrações em vários ambientes e podemos classificá-las pelo modo como são transmitidas ao corpo: vibração de corpo inteiro e vibração transmitida por meio das mãos. A vibração de corpo inteiro ocorre quando o corpo está sendo suportado por uma superfície que vibra. É produzida de três formas: ao sentarmos num assento que vibra, ao ficarmos em pé num piso que vibra, ou ao se deitarmos numa superfície vibrante. Esse tipo de vibração ocorre em todas as formas de transporte.

A vibração também pode ser classificada como sendo transmitida por meio das mãos. Esta é produzida por diversos processos na indústria, agricultura, mineração e construção, quando equipamentos vibratórios são manipulados pelos dedos ou mãos (Griffin, 1982 apud Carnicelli, 1994). Em geral, a vibração típica dos equipamentos é maior do que $0,316 \mathrm{~m} / \mathrm{s}^{2}$ (Matoba, 1994), enquanto o nível de pressão sonora é geralmente maior que $95 \mathrm{~dB}(\mathrm{~A})$. A ação repetida desses estressores no corpo humano pode sobrecarregar e prejudicar não somente o sistema nervoso periférico, mas também o sistema nervoso central.

Independentemente de como é transmitida a vibração, é preciso conhecer seus efeitos para que, além da prevenção, possamos fazer o diagnóstico diferencial entre doenças com sintomas semelhantes, decorrentes da exposição a vibrações ou distúrbios osteoarticulares relacionados ao trabalho (DORT). Há uma grande variação entre indivíduos quanto a sua capacidade de perceber a vibração e de considerá-la desconfortável ou inaceitável. O indivíduo precisa estar exposto a vibração há vários anos para que ocorram mudanças em seu estado de saúde.

Na norma ISO 2631 (1997), consta que a exposição a vibração de corpo inteiro causa uma complexa distribuição de movimentos oscilatórios e forças dentro do organismo. Pode existir grande variação de queixas e intensidade delas em relação aos efeitos biológicos por ela causados. A vibração pode causar sensação de desconforto e mal humor, influenciar o desempenho ou oferecer risco à saúde e segurança.

Para Pekkarinen (1995), a vibração de corpo inteiro é um estímulo difuso que excita vários receptores simultaneamente e causa estresse geral. Os efeitos da vibração de corpo inteiro estão bem proximamente relacionados aos efeitos do ruído de baixa freqüência. $\mathrm{O}$ autor relata que a vibração de corpo inteiro tem sido responsabilizada por alterações na circulação sangüínea da orelha interna e que tem sido observado uma redução 
temporária do limiar auditivo entre nas freqüências de 2 e 4 $\mathrm{kHz}$, ligada à vibração de corpo inteiro.

Matoba (1994) refere que dor de cabeça, insônia, esquecimento, irritabilidade, depressão, zumbido e impotência aparecem em indivíduos expostos à vibração através das mãos à medida que os sinais e sintomas vão progredindo. Entretanto as alterações mais comuns seriam da circulação periférica, nervosa e muscular, da articulação e do sistema nervoso central e autônomo, associada com perda auditiva nistagmo e vertigem. Essas alterações são observadas em 60 a 70\% dos pacientes. A avaliação física revela sinais de alterações circulatórias, nervosas e musculares nos dedos e braços.

\section{Ruído e Vibração}

A ação da exposição combinada aos riscos ruído e vibração pode ocasionar um efeito sinérgico à saúde dos trabalhadores. Mannimen (1984) apud Castaño e Fernandez (1989) descreve um aumento sistemático do estresse e outros efeitos deletérios em trabalhadores expostos à ação combinada de ruído e vibrações em geral em relação a trabalhadores expostos a um ou outro risco isoladamente.

Vários estudos relatam que trabalhadores que sofriam da síndrome do dedo branco desenvolveram maior perda auditiva que o grupo controle (Pyykko et al., 1981, 1994; Axelsson et al., 1989; Iki, 1994; Miyakita et al., 1997). Eles referem que o mecanismo mais provável da perda auditiva induzida por níveis de pressão sonora elevados é a vasoconstrição do ouvido interno, causada pela exposição ao ruído e pelo aumento da demanda de oxigênio para fortalecer e prolongar a excitação dos receptores das células que aumentam o risco isquêmico das células ciliadas.

Murata, Araki e Aono (1990) examinaram os efeitos de vibração através das mãos no sistema nervoso central e periférico por meio do exame do potencial auditivo evocado. Os autores concluíram que a exposição combinada a estressores como vibrações, ruído, diferenças climáticas e trabalho pesado, afeta não apenas o sistema nervoso periférico, mas também o sistema nervoso central.

\section{MATERIAL E MÉTODO}

Segundo o Departamento de Saúde Ocupacional da empresa estudada, o nível de pressão sonora a que os dois grupos encontram-se expostos é o mesmo, de aproximadamente $92 \mathrm{~dB}(\mathrm{~A})$. Por indisponibilidade de equipamentos adequados, a vibração a que esses trabalhadores estão submetidos não foi medida, mas encontra-se descrita abaixo.

Todos os trabalhadores dessa empresa se submetem a exame audiométrico periódico anualmente ou a critério do médico do trabalho. Como este trabalho é um estudo epidemiológico transversal, os exames anteriores não foram considerados. Todos os trabalhadores já foram submetidos ao exame audiométrico pelo menos uma vez, portanto estão familiarizados com o procedimento do teste. Os trabalhadores foram avaliados na Clínica de Fonoaudiologia da Universidade Tuiuti do Paraná.

Durante a entrevista obtivemos dados sobre a rotina de trabalho desses trabalhadores. Os trabalhadores do Grupo 1 têm como rotina de trabalho a preparação do equipamento, que inclui o abastecimento de combustível para o seu funcionamento. Esse combustível dura em média 45 minutos, e o trabalhador é obrigado a interromper sua atividade para reabastecer o equipamento e lixar/afiar as lâminas cortadoras. Esse processo leva em média 15 minutos. Na segunda parada, tanto no turno da manhã quanto no da tarde, os trabalhadores também fazem um lanche, o que estende a duração da pausa.

Essa rotina não é compartilhada pelos trabalhadores do Grupo 2. Não são feitas pausas durante seu período de trabalho. A rotina dos trabalhadores do Grupo 2 é a de ligar o equipamento e mantê-lo funcionando até o horário do almoço, no período da manhã, e até o final do expediente no período da tarde.

\section{Caracterização dos sujeitos}

Os trabalhadores avaliados trabalham numa empresa de conservação e limpeza de vias públicas da região metropolitana de Curitiba, em regime de turno fixo, das $7 \mathrm{~h}$ às 17h30. O tempo mínimo de exposição ao ruído ocupacional é de quatro horas diárias há pelo menos um ano. Todos encontravam-se no momento da avaliação audiológica em repouso acústico de no mínimo 14 horas, não apresentavam perfuração de membrana timpânica, obstrução do meato acústico externo e nem exposição extraocupacional ao ruído e/ou vibração.

Os 73 trabalhadores avaliados foram divididos em dois grupos em função da forma como a vibração lhes é transmitida ao corpo: Grupo 1 - vibração transmitida por meio das mãos e braços - em 38 trabalhadores que operam motorroçadeiras, e Grupo 2 - vibração de corpo inteiro em 35 trabalhadores que operam motoniveladora, pá carregadeira, rolo compressor e retroescavadeira. Na Tabela 1 encontra-se a idade média, o tempo de exposição ao ruído em empregos anteriores e o tempo de exposição total ao ruído, ou seja, em toda a vida laboral dos sujeitos examinados.

Pode-se observar que os dois grupos são bastante semelhantes na média de idade, e a exposição ocupacional é bastante homogênea no que se refere ao tempo de exposição anterior, atual e total. Porém, em relação ao tempo de exposição diária, há uma diferença pequena quanto ao número de horas de exposição entre os dois grupos. Os trabalhadores do Grupo 1 têm uma média diária de exposição de 6 horas e os do Grupo 2, de 7 horas. Ambas ultrapassam o número máximo de exposição diária para esta intensidade (92 dBA), preconizada pela Norma Regulamentadora $\mathrm{n}$ o 15 (NR 15) do Ministério do Trabalho (Brasil, 1998), que seria de no máximo 4 horas diárias e pela American Conference 
Tabela 1. Descrição dos participantes do estudo, segundo idade e exposição ocupacional a ruído, no emprego atual e em empregos anteriores

\begin{tabular}{|c|c|c|c|c|}
\hline \multirow{2}{*}{ Variável } & \multicolumn{2}{|c|}{ Grupo 1} & \multicolumn{2}{|c|}{ Grupo 2} \\
\hline & Média & (Mín/Máx.) & Média & (Mín/Máx.) \\
\hline Idade (em anos) & 41.1 & $(29-49)$ & 9.9 & $(31-50)$ \\
\hline Tempo de exposição total (em anos) & 14.5 & $(1-26)$ & 12.7 & $(8-40)$ \\
\hline Tempo de exposição atual (em anos) & 12.3 & $(1-23)$ & 12.4 & $(4-26)$ \\
\hline Tempo de exposição anterior (em anos) & 2.2 & $(0-20)$ & 0.3 & $(0.5-25)$ \\
\hline Tempo de exposição diária (em horas) & 6.1 & $(4-8)$ & 7.2 & $(4-10)$ \\
\hline
\end{tabular}

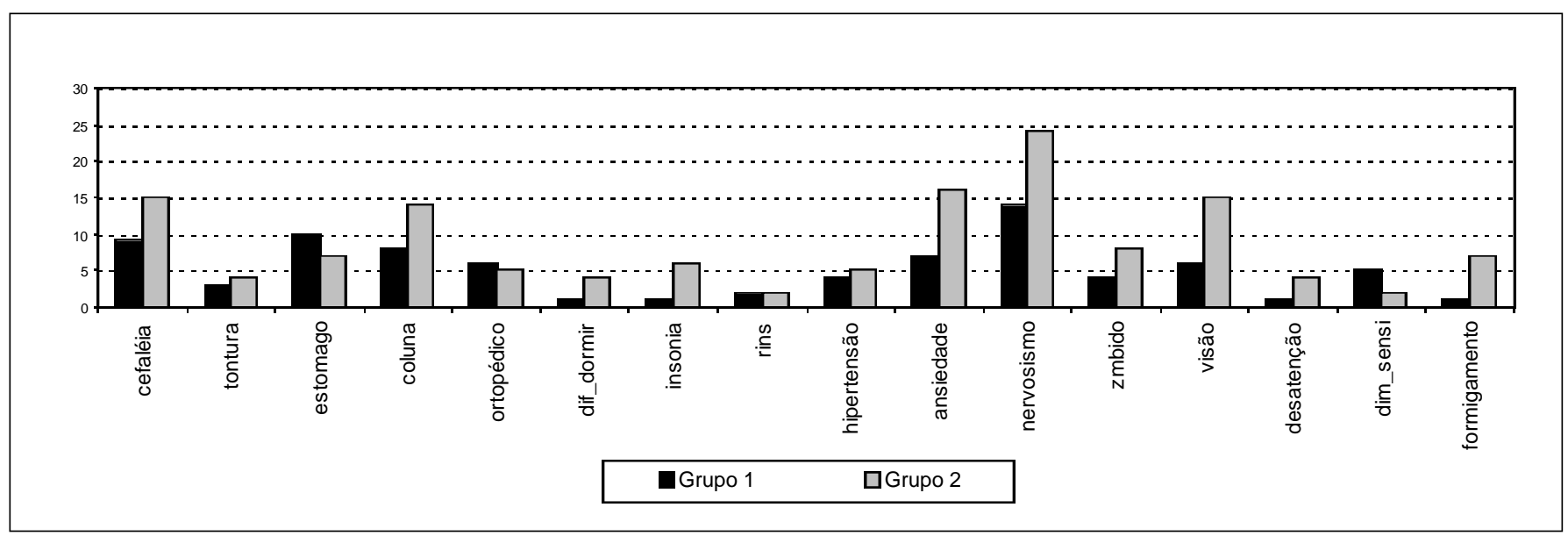

Gráfico 1. Número de queixas de saúde apresentadas pelos participantes do estudo, por grupo de exposição

of Governmental Industrial Hygienists (ACGIH), que seria de no máximo duas horas diárias.

Roteiro de entrevista

Os tópicos do roteiro de entrevista incluem: antecedentes ocupacionais de exposição a níveis elevados de pressão sonora ocupacional, exposição não-ocupacional ao mesmo agente, antecedentes de patologias auditivas, antecedentes mórbidos com possíveis implicações auditivas, uso de medicamentos e sua ação sobre a audição, uso de protetor auditivo e sua freqüência (ver Anexo 1). Essa entrevista foi aplicada também com o objetivo de levantar as queixas auditivas e extra-auditivas dos participantes.

\section{Avaliação audiológica}

A audiometria foi realizada em cabina com isolamento acústico, com audiômetro Maico MA 41 e fone TDH 39, todos submetidos a calibração anual segundo padrão ISO R 389 (1998) para via aérea e ISO 7566 (1987) para via óssea. Diariamente foi realizada a calibração biológica, com o intuito de identificar a ocorrência de algum problema de calibração no aparelho.

\begin{abstract}
RESULTADOS
Entre os trabalhadores do Grupo 1 as queixas mais comuns foram nervosismo, problemas de estômago, e problemas de coluna e entre os trabalhadores do Grupo 2, foram nervosismo, ansiedade, cefaléia e problemas visuais, problemas de coluna, zumbido, e problemas de estômago e formigamento ou esbranquiçamento dos dedos. A ocorrência de queixas foi semelhante entre os dois grupos, porém com uma freqüência maior nos trabalhadores do Grupo 2 (dor de cabeça, problemas de coluna, dificuldade para dormir, insônia, nervosismo, zumbido, problemas de visão, desatenção e formigamento dos dedos). As exceções foram as queixas de diminuição da sensibilidade cutânea e problemas de estômago e ortopédicos.

Os resultados da audiometria foram analisados segundo o critério de classificação utilizado por Fiorini (1994). Esse critério classifica os achados nas seguintes categorias: normal (todos os limiares iguais ou inferiores a 25dBNA), normal com entalhe (rebaixamento numa das freqüências de 3,4 ou $6 \mathrm{kHz}$, com diferença de pelo menos $10 \mathrm{~dB}$ em relação a freqüência anterior ou posterior) e traçado audiométrico sugestivo de perda auditiva induzida
\end{abstract}


Anexo 1. Roteiro de entrevista

Nome: (iniciais)

Idade: Escolaridade:

Data:

1- Qual sua profissão?

2- Trabalha com equipamento barulhento? ( ) não ( ) sim. Qual?

3- Quantas horas por dia?

4- Há quanto tempo?

5- Sente algum mal estar após trabalhar com este equipamento? ( ) não ( ) sim. Há quanto tempo?

6- Antes deste trabalho atual você trabalhou em algum lugar muito barulhento?

( ) não ( ) sim. Por quanto tempo?

7- Mora ou freqüenta lugares barulhentos onde precise falar alto para poder conversar?

( ) $\operatorname{sim}($ ) não

8- Você pratica ou praticou tiro ou alvo? ( ) $\operatorname{sim}($ ) não

9- Serviu o exército?

( ) $\operatorname{sim}($ ) não

10- Freqüenta discoteca ou locais onde tem música amplificada? ( ) não ( ) sim. ( ) uma vez por semana

() uma vez por mês ( ) uma vez por ano

11- Pratica alguma atividade barulhenta fora do expediente de trabalho? ( ) não ( ) sim. Qual?

Com que freqüência?

12- Faz uso de protetor auditivo?( ) não

( ) $\operatorname{sim}($ ) de concha

( ) de inserção

( ) sim, de vez em quando

( ) quase sempre que exposto a barulho alto

( ) sempre que exposto à barulho alto

13- Ouve bem?( ) sim

( ) não ( ) ouvido D ( ) ouvido E ( ) ambos

14- Entende bem o que as pessoas falam? ( ) sim ( ) não

15- Tem dor de ouvido?( ) não ( ) sim ( ) D ( ) E Quando

16- Tem zumbido?( ) não ( ) sim ( ) direito ( ) esquerdo ( ) ambos Com que freqüência?

Desde quando?

Em que momento?

Esse zumbido é: ( ) grosso ( ) fino ( ) apito ( ) chuva

17- Alguma pessoa na família tem problema auditivo? ( ) sim ( ) não Qual o grau de parentesco?

18- Sente dor de cabeça? ( ) sim ( ) não

19- Tontura? ( ) sim ( ) não

20- Problema de estômago? ( ) sim ( ) não

21- Problema de rins? ( ) sim ( ) não
22- Problema de coluna? ( ) sim ( ) não

23- Você tem algum problema ortopédico?

( ) não ( ) sim. Qual?

24- Sente dificuldade para dormir? ( ) sim ( ) não

25- Tem insônia? ( ) sim ( ) não

26- É desatento? ( ) sim ( ) não

27- É nervoso? ( ) sim ( ) não

28- É ansioso? ( ) sim ( ) não

29- É depressivo? ( ) sim ( ) não

30- Enxerga bem? ( ) sim ( ) não

31- Tem problema de pressão?

Alta ( ) sim () não Baixa ( ) sim ( ) não

32- Tem taquicardia?( ) sim Quando? ( ) não

33- Algum problema de sensibilidade cutânea? ( ) sim ( ) não

34- Você percebe esbranquiçamento dos dedos? ( ) sim ( ) não ( ) durante o trabalho ( ) após o trabalho

35- 0 médico mandou você tomar algum remédio para algum desses problemas? ( ) não ( ) sim.Qual remédio? Para qual problema?

36- Em média, quantos copos de bebida alcoólica você bebe?

( ) mais de seis por dia

() de 3 a 5 por dia

() de 1 a 3 por dia

( ) de 3 a 4 por semana

( ) nunca

37- Fuma? ( )sim ( ) não

Quanto?

Há quanto tempo?

38- Fica doente com freqüência? ( )sim ( ) não

39- Quando procurou um médico pela última vez?(mês/ano)

40- Qual motivo?

41- Foi receitado algum medicamento?
( ) não
( ) sim Qual?

42- Você usou o remédio como foi receitado? ( )sim ( ) não. Por quê?

43- Você continua tomando o remédio?

( ) sim.Com que freqüência?

( ) não. Por quanto tempo tomou?

44- Sente algum problema de saúde que não tenha sido mencionado? ( ) não ( ) sim. Qual?

45- Está usando algum medicamento no momento? ( ) não ( ) sim. Qual? Por que?

46- Você percebeu alguma mudança na sua audição depois de alguma doença séria?

( ) não ( ) sim. Qual doença?

47- Você notou alguma diferença na sua audição depois que tomou remédio? ( ) não ( ) sim ( ) melhorou ( ) piorou 
pelo ruído (PAIR) ou seja, configuração de PAIR, mas ainda limiares auditivos acima de $25 \mathrm{dBNA}$ na faixa de freqüência de 3 a $6 \mathrm{kHz}$.

$\mathrm{Na}$ Tabela 2, são encontradas as classificações audiométricas, de acordo com o tempo de exposição. Observa-se na Tabela 3 que as curvas audiométricas com entalhe prevalecem entre 11-26 anos de exposição ao ruído no grupo 1.

No Grupo 1, a maior porcentagem de audiometrias normais (50\%) ocorreu em trabalhadores expostos entre 0 e 10 anos. Essa porcentagem é menor à medida que aumenta o tempo de exposição em ambos os grupos, porém de forma mais acentuada no Grupo 1. É evidente que há mais casos de PAIR a partir de dez anos de exposição.

Todos os trabalhadores do Grupo 1, sem exceção, referiram uso de protetor auricular, sendo que 13 usam o tipo concha e 11 o tipo plug. Um trabalhador referiu usar o protetor de vez em quando, 2 quase sempre, e 21 sempre que expostos a barulho alto, ou seja, quando o equipamento está ligado. Mesmo com o uso do protetor auricular, foram observados 6 casos de PAIR.

Em relação ao uso de protetores auriculares entre os trabalhadores do Grupo 2, 9 trabalhadores com audição alterada admitiram que não os usam. Entre os 12 que referiram uso, 7 utilizam o tipo concha e 5 o tipo plug; 4 os utilizam de vez em quando, 1 faz uso quase sempre e 7 afirmaram fazer uso do protetor auricular sempre que expostos a barulho alto, ou seja, sempre que estão com o equipamento funcionando. É maior o número de trabalhadores que usam o protetor e apresentam limiares auditivos normais no grupo 1 .

Os trabalhadores do Grupo 1, sem exceção, fazem uso do protetor auditivo e apenas 4 (11\%) referiram zumbido. No Grupo 2 é maior o número de trabalhadores com zumbido que não usam o protetor auditivo que o número dos que usam. Dos 35 trabalhadores, 14 não usam protetor auditivo e 6 (43\%) apresentaram zumbido; 21 usam protetor auditivo e 3 (14\%) têm zumbido. Do total de trabalhadores investigados, 59 usam protetor auditivo, apenas sete (10\%) referiram zumbido, 14 não usam protetor e entre eles 6 (8\%) apresentaram zumbido. Oito (11\%), mesmo não usando protetor, não referiram zumbido.

Não foi observada uma tendência de associação entre a queixa de formigamento de dedos e alterações auditivas.

Tabela 2. Número e porcentagem das classificações audiométricas dos participantes, de acordo com o grupo e tempo de exposição

\begin{tabular}{|c|c|c|c|c|c|c|c|c|c|c|c|c|c|c|c|c|c|c|c|c|}
\hline \multirow{3}{*}{$\begin{array}{l}\text { EXPOSIÇÃO } \\
\text { (em anos) }\end{array}$} & \multicolumn{8}{|c|}{ GRUPO 1} & \multicolumn{12}{|c|}{ GRUPO 2} \\
\hline & \multicolumn{2}{|c|}{ Normal } & \multicolumn{2}{|c|}{ Entalhe } & \multicolumn{2}{|c|}{ PAIR (uni) } & \multicolumn{2}{|c|}{ PAIR (bi) } & \multicolumn{2}{|c|}{ Total } & \multicolumn{2}{|c|}{ Normal } & \multicolumn{2}{|c|}{ Entalhe } & \multicolumn{2}{|c|}{ PAIR (uni) } & \multicolumn{2}{|c|}{ PAIR (bi) } & \multicolumn{2}{|c|}{ Total } \\
\hline & $\mathrm{n}$ & $\%$ & $\mathrm{n}$ & $\%$ & $\mathrm{n}$ & $\%$ & $\mathrm{n}$ & $\%$ & $n$ & $\%$ & $\mathrm{n}$ & $\%$ & $\mathrm{n}$ & $\%$ & $\mathrm{n}$ & $\%$ & $\mathrm{n}$ & $\%$ & $\mathrm{n}$ & $\%$ \\
\hline $0-10$ & 9 & 50 & 6 & 33 & 2 & 11 & 1 & 5 & 18 & 100 & 8 & 50 & 5 & 31 & 1 & 6 & 2 & 12 & 16 & 100 \\
\hline $11-26$ & 5 & 25 & 8 & 40 & 2 & 10 & 5 & 25 & 20 & 100 & 8 & 42 & 3 & 16 & 2 & 10 & 6 & 32 & 19 & 100 \\
\hline
\end{tabular}

Tabela 3. Resultados dos achados audiométricos em relação ao uso de protetor auditivo, de acordo com o grupo de exposição

\begin{tabular}{|c|c|c|c|c|c|c|c|c|c|c|c|c|c|c|}
\hline \multirow[t]{3}{*}{$\begin{array}{l}\text { IDADE } \\
\text { (EM ANOS) }\end{array}$} & \multicolumn{4}{|c|}{$\begin{array}{c}\text { GRUPO } 1 \\
\text { PROTETOR }\end{array}$} & \multicolumn{4}{|c|}{$\begin{array}{l}\text { GRUPO } 2 \\
\text { PROTETOR }\end{array}$} & \multicolumn{4}{|c|}{$\begin{array}{c}\text { TOTAL } \\
\text { PROTETOR }\end{array}$} & \multirow{2}{*}{\multicolumn{2}{|c|}{ TOTAL }} \\
\hline & \multicolumn{2}{|c|}{ Sim } & \multicolumn{2}{|c|}{ Não } & \multicolumn{2}{|c|}{ Sim } & \multicolumn{2}{|c|}{ Não } & \multicolumn{2}{|c|}{$\operatorname{Sim}$} & \multicolumn{2}{|c|}{ Não } & & \\
\hline & $\mathrm{n}$ & $\%$ & $\mathrm{n}$ & $\%$ & $\mathrm{n}$ & $\%$ & $\mathrm{n}$ & $\%$ & $\mathrm{n}$ & $\%$ & $\mathrm{n}$ & $\%$ & $\mathrm{n}$ & $\%$ \\
\hline Normal & 14 & 37 & 0 & 0 & 11 & 31 & 5 & 14 & 25 & 34 & 5 & 7 & 30 & 41 \\
\hline Alterados & 24 & 63 & 0 & 0 & 10 & 29 & 9 & 26 & 34 & 47 & 9 & 2 & 43 & 59 \\
\hline Total & 38 & 100 & 0 & 0 & 21 & 60 & 14 & 40 & 59 & 81 & 14 & 19 & 73 & 100 \\
\hline
\end{tabular}

Tabela 4. Relação entre queixa de zumbido e uso de protetor auditivo, de acordo com o grupo de exposição

\begin{tabular}{|c|c|c|c|c|c|c|c|c|c|c|c|c|c|c|}
\hline \multirow[t]{3}{*}{ ZUMBIDO } & \multicolumn{4}{|c|}{$\begin{array}{r}\text { GRUPO } 1 \\
\text { PROTETOR }\end{array}$} & \multicolumn{4}{|c|}{$\begin{array}{l}\text { GRUPO } 2 \\
\text { PROTETOR }\end{array}$} & \multicolumn{4}{|c|}{$\begin{array}{c}\text { TOTAL } \\
\text { PROTETOR }\end{array}$} & \multicolumn{2}{|c|}{ TOTAL } \\
\hline & \multicolumn{2}{|c|}{ Sim } & \multicolumn{2}{|c|}{ Não } & \multicolumn{2}{|c|}{ Sim } & \multicolumn{2}{|c|}{ Não } & \multicolumn{2}{|c|}{ Sim } & \multicolumn{2}{|c|}{ Não } & & \\
\hline & $\mathrm{n}$ & $\%$ & $\mathrm{n}$ & $\%$ & $\mathrm{n}$ & $\%$ & $\mathrm{n}$ & $\%$ & $\mathrm{n}$ & $\%$ & $\mathrm{n}$ & $\%$ & $\mathrm{n}$ & $\%$ \\
\hline Sim & 4 & 11 & 0 & 0 & 3 & 9 & 6 & 17 & 7 & 10 & 6 & 8 & 13 & 18 \\
\hline Não & 34 & 89 & 0 & 0 & 18 & 51 & 8 & 23 & 52 & 71 & 8 & 11 & 60 & 82 \\
\hline Total & 38 & 100 & 0 & 0 & 21 & 60 & 14 & 40 & 59 & 81 & 14 & 19 & 73 & 100 \\
\hline
\end{tabular}


Tabela 5. Classificações audiométricas em relação às queixas de formigamento e esbranquiçamento dos dedos, por grupo de exposição

\begin{tabular}{|c|c|c|c|c|c|c|c|c|c|c|c|c|}
\hline \multirow{4}{*}{$\begin{array}{l}\text { CLASSIFICAÇÃO } \\
\text { AUDIOMÉTRICA }\end{array}$} & \multicolumn{6}{|c|}{ GRUPO 1} & \multicolumn{6}{|c|}{ GRUPO 2} \\
\hline & \multicolumn{6}{|c|}{ FORMIGAMENTO/ESBRANQUIÇAMENTO } & \multicolumn{6}{|c|}{ FORMIGAMENTO/ESBRANQUIÇAMENTO } \\
\hline & \multicolumn{2}{|c|}{ SIM } & \multicolumn{2}{|c|}{ NÃO } & \multicolumn{2}{|c|}{ TOTAL } & \multicolumn{2}{|c|}{ SIM } & \multicolumn{2}{|c|}{ NÃO } & \multicolumn{2}{|c|}{ TOTAL } \\
\hline & $\mathrm{n}$ & $\%$ & $\mathrm{n}$ & $\%$ & $\mathrm{n}$ & $\%$ & $\mathrm{n}$ & $\%$ & $\mathrm{n}$ & $\%$ & $\mathrm{n}$ & $\%$ \\
\hline Normal & 3 & 22 & 11 & 78 & 14 & 100 & 3 & 19 & 13 & 81 & 16 & 100 \\
\hline Alterada & 3 & 12 & 21 & 88 & 24 & 100 & 5 & 26 & 14 & 74 & 19 & 100 \\
\hline Total & 6 & 16 & 32 & 84 & 38 & 100 & 8 & 23 & 27 & 77 & 35 & 100 \\
\hline
\end{tabular}

\section{DISCUSSÃO}

Queixas extra-auditivas

As queixas mais freqüentes foram nervosismo, ansiedade, cefaléia, zumbido e problemas de estômago. Esse achado confirma as observações publicadas anteriormente por Bevilacqua et al. (1987) e Andrade et al. (1998). As queixas de hipertensão arterial corroboram com o relato de Griffin (1982) e Axelsson (1989), e a queixa de formigamento e esbranquiçamento dos dedos está de acordo com Carnicelli (1994) e Okada (1990), enquanto os problemas de visão e diminuição da sensibilidade cutânea confirmam as observações de Gerges (1997). Problemas de coluna, ortopédicos e desatenção também haviam sido reportados por Griffin (1982) apud Carnicelli (1994) e Matoba (1994).

A diferença nas porcentagens de queixas de saúde entre os dois grupos sugere que a exposição a vibração de corpo inteiro e a rotina de trabalho sem pausas podem afetar a saúde dos trabalhadores de forma mais freqüente e acentuada, observação também encontrada na literatura em Pekkarinen (1995) e ISO 2631 que afirmam que os efeitos da vibração de corpo inteiro na saúde aplicam-se primariamente a pessoas sentadas, uma vez que os efeitos da vibração em pessoas em pé, reclinadas ou recostadas são pouco conhecidos. Essa observação indica a necessidade que medidas sejam propostas para redução dessa exposição.

Queixas auditivas e achados audiométricos

No Grupo 2, o número de trabalhadores que se queixam de zumbido foi duas vezes maior que no Grupo 1. Esse fato talvez possa ser explicado pelo uso diário do protetor auditivo por todos os trabalhadores do Grupo 1 durante todo o período em que o equipamento está funcionando. No Grupo 2, apenas 21 ou 60\% dos trabalhadores usam protetor auditivo e, mesmo assim, somente de vez em quando. Entre os que fazem uso do protetor, 3 (14\%) queixaram-se de zumbido. As justificativas mais encontradas para esse comportamento nos trabalhadores do Grupo 2 são que os protetores esquentam muito as orelhas, e que eles necessitam da audição para avaliar e monitorar o funcionamento do equipamento.
Observou-se que entre os 11\% dos trabalhadores do Grupo 1 que apresentaram queixa de zumbido, 5\% apresentaram resultado de audiometria normal. No grupo 2 , 23\% queixaram-se de zumbido; $11 \%$ apresentaram o resultado de audiometria normal. Este dado não foi o mesmo observado por Bevilacqua et al. (1987), Matoba (1994) e Andrade et al. (1998) que encontram zumbido mais comumente associado à PAIR.

Hempstock e O'Connor (1978) demonstraram que quando há interrupção da exposição à vibrações durante o dia o resultado pode ser benéfico quanto a seus efeitos no trabalhador. Os autores observaram também que antes da introdução do dispositivo de antivibração nas serras elétricas, os trabalhadores apresentavam os primeiros sinais da síndrome do dedo branco depois de um período de latência de três anos. Depois da introdução do dispositivo de antivibração, houve uma melhora evidente dos sintomas. Da mesma forma, intervalos durante exposição à ruído também reduzem o tempo total de exposição e o risco que ela representa.

Uma observação que está de acordo com a constatação de Ferraz (1995) foi a de que muitos trabalhadores não associam as queixas como sendo oriundas de suas atividades profissionais. Em alguns trabalhadores observou-se também receio de relatar suas queixas e problemas de saúde e sofrer alguma retaliação no trabalho por parte da empresa como, por exemplo, a transferência para uma outra função em que não haja o percentual de gratificação por se estar submetido a algum risco ocupacional e a discriminação por parte dos companheiros de trabalho.

Segundo Zhu et al. (1997), os efeitos combinados de ruído e vibrações transmitida por meio das mãos-braços podem aumentar a ocorrência de perda auditiva, mas também para Pekkarinen (1995) esse aumento pode estar associado à susceptibilidade individual e supostamente ao sistema nervoso autônomo que regula a reação da vascularização periférica.

Em relação ao formigamento e esbranquiçamento dos dedos, dos 6 (17\%) trabalhadores do Grupo 1 que apresentaram essa queixa, 3 apresentaram resultado de audiometria normal, um, entalhe unilateral, um, entalhe bilateral e um, PAIR bilateral. No Grupo 2, 8 (23\%) apresentaram a queixa; 3 apresentaram resultado de 
audiometria normal, 1 entalhe unilateral, 1 PAIR unilateral e 3 PAIR bilateral. Esses dados, portanto, não coincidem com os encontrados por Starck et al. (1988) em seus estudos nos quais trabalhadores que apresentavam sintomas da síndrome do dedo branco tinham limiares auditivos 10 dBNA piores dos que os que não apresentavam sintomas. Há que se levar em consideração que nesta pesquisa está sendo considerado a queixa e não o sintoma como um provável diagnóstico da doença propriamente dita. Além disso, o tamanho desta amostra pode ter sido insuficiente para permitir a observação desses efeitos relatados pela literatura.

\section{Medidas preventivas}

Para prevenção de perdas auditivas no trabalho, há concordância por inúmeros autores de que o controle da exposição deve ser sempre a primeira alternativa a ser considerada (NIOSH, 1996). Entretanto, por dificuldades técnicas e econômicas, Gerges (1999) argumenta que o uso de protetores auriculares é a medida mundialmente adotada e difundida por ser pouco dispendiosa e de fácil acesso. O National Institute for Occupational Safety and Health (NIOSH,1998) afirma que a atenuação que um protetor auditivo oferece depende de suas características e de como o trabalhador o utiliza. O protetor selecionado deve ser capaz de manter a exposição auditiva ao ruído abaixo de $85 \mathrm{~dB}(\mathrm{~A})$. Os trabalhadores e supervisores devem certificar-se periodicamente de que o protetor está sendo usado corretamente, que está bem ajustado e que é apropriado ao ruído ao qual o trabalhador está sujeito. A ênfase tem sido dada à atenuação que o protetor oferece e outras qualidades necessárias para a sua efetividade têm sido negligenciadas. As pessoas que fazem a seleção do protetor auricular devem considerar para que tipo de ruído ele será usado e quem irá usá-lo, a necessidade de compatibilidade dele com outros equipamentos de proteção, conforto, e as condições do local de trabalho, como temperatura, umidade e pressão atmosférica.

O uso intermitente dos protetores auriculares reduz dramaticamente sua efetividade. Um protetor que atenua 30 dB(A) em oito horas de exposição atenuará apenas 15 $\mathrm{dB}(\mathrm{A})$ se o trabalhador deixar de usá-lo por um período cumulativo de 30 minutos durante um dia de oito horas de trabalho (NIOSH, 1998).

As medidas adotadas pela empresa estudada restringem-se à oferta de protetores auditivos, sem controle sobre o uso. A periodicidade dos exames audiométricos é decidida pelo médico e acontece entre uma vez por ano e uma vez a cada três anos segundo o relato dos trabalhadores. Estes relataram também que alguns colegas de trabalho foram transferidos de suas funções por apresentarem rebaixamento auditivo. Não existe um programa de prevenção de perdas auditivas estruturado para a população do estudo, apesar de a necessidade ser evidente. Recomendações detalhadas para programas de prevenção de perdas auditivas relacionadas ao trabalho podem ser encontradas nos documentos do NIOSH
(1996, 1998). Tais documentos podem ser acessados em sua integra, pela Internet nos endereços http://www.cdc.gov/ niosh/96-110.html e http://www.cdc.gov/niosh/98-126.html. Algumas recomendações para tais programas podem ser encontradas em português em Ferraz (1995).

No manual de uma das motorroçadeiras usadas, foram encontradas algumas orientações sobre como se minimizar os efeitos da vibração, limites de exposição (TLVs) segundo a ACGIH (1999) apud Associação Brasileira de Higienistas Ocupacionais (1999) e segundo o NIOSH (1989) no documento Criteria for a Recommended Standard Occupational Exposure to Hand-Arm Vibration.

$\mathrm{Na}$ população estudada neste trabalho, observa-se apenas o uso de alguns equipamentos de proteção individual (óculos, protetor auricular) como medida de prevenção dos efeitos da vibração. Porém, é notória a necessidade de aplicação dessas medidas e da priorização de um treinamento do trabalhador que enfatize procedimentos como os sugerido no Criteria for a Recommended Standard Occupational Exposu re to Hand-Arm Vibration (NIOSH, 1989):

- reconhecer sinais e sintomas decorrentes de vibrações;

- registrar todos os sinais e sintomas;

- criar norma para supervisão médica na prevenção e controle das doenças decorrestes de vibrações;

- levantar os possíveis efeitos na saúde por se continuar operando equipamentos vibrantes;

- saber da reversibilidade quando detectados precocemente sinais e sintomas;

- criar norma para produção e manutenção de equipamentos vibrantes;

- observar aspectos ergonômicos do uso do equipamento;

- saber da necessidade de aquecimento e conhecer procedimentos para manter o corpo e as mãos aquecidas e secas;

- usar de roupas e equipamentos de proteção;

- realizar medições dos agentes de risco;

- estabelecer horário de trabalho e descanso para controlar a duração da exposição;

- informar ao supervisor de qualquer funcionamento anormal dos equipamentos;

- estar ciente da possibilidade de agravamento da síndrome produzida pela vibração por meio das mãos-braços com o hábito de fumar e o uso de algumas drogas.

\section{CONCLUSÕES}

Este estudo avaliou as queixas de saúde e achados audiológicos em trabalhadores expostos a ruído excessivo e vibração mãos-braços versus corpo inteiro. Em relação aos problemas de saúde os trabalhadores expostos a vibração corpo inteiro foram os que apresentaram um maior número de queixas 
que incluíram : cefaléia, tontura, problemas de coluna, problemas de sono, hipertensão, ansiedade, nervosismo, desatenção, formigamento e esbranquiçamento dos dedos, zumbido e problemas de visão. Entretanto, a porcentagem de audiogramas alterados foi mais elevada no grupo exposto a vibração transmitida por meio das mãos-braços.

Concluiu-se que há necessidade da implantação de medidas para a medição e o controle da exposição à vibração e ruído. O elevado número de queixas de saúde indica a necessidade da aplicação de outros exames que caracterizem ou diagnostiquem efetivamente os sintomas decorrentes da exposição a vibrações. Este estudo também evidenciou a necessidade de orientação tanto para a direção da empresa quanto para os próprios trabalhadores em relação à implementação de programas de prevenção de doenças ocupacionais que contemplem também o programa de prevenção de perdas auditivas.

O estudo sugere a realização de outras pesquisas para avaliação da adequação dos atuais limites de exposição quando há exposição continuada a ruído e vibração.

\section{AGRADECIMENTOS}

Agradecemos a Dra. Dóris Ruth Lewis e Professor Dr. Luiz Gonzaga Calefe pelo apoio na fase inicial deste estudo, ao IMAP - Instituto Municipal de Administração Pública (Curitiba) pela bolsa de estudo concedida, e aos trabalhadores que concordaram em participar deste estudo.

\section{REFERÊNCIASBIBUOGRÁFICAS}

1. Andrade DR, Marini AL, Finkler C, Capp E, Closs M. Efeitos do ruído industrial no organismo. Pró-Fono, 10 (1):17-20, 1998.

2. American Conference of Governmental Industrial Hygienists (Acgih) Associação Brasileira de Higienistas Ocupacionais. Limites de exposição para substâncias químicas e agentes físicos e índices biológicos de exposição. Trad. ABHO. Campinas, 1999.

3. Axelsson A, Hamernik RP, Ahroon WA. Noise and vibration interactions: effects on hearing. J Acoust Soc Am 1989;86(6):2129-37.

4. Bevilacqua MC, Lewis DR, Morata TC. Programas de conservação auditiva. Dist Com 1987;3-4(2):143-51.

5. Brasil, Norma Regulamentadora 15. Limites de tolerância para ruído contínuo ou intermitente. Portaria $\mathrm{n}^{\mathrm{O}} 3.214$, de 8 de junho de 1978. In: Segurança e Medicina do Trabalho, 16:123-34, São Paulo: Atlas; 1998.

6. Brasil, Ministério De Trabalho, Secretaria de Segurança e Saúde do Trabalho, Portaria N.․ 19, 1998.

7. Carnicelli MVF. Exposição ocupacional à vibração transmitida através das mãos: uma revisão sobre o distúrbio vascular periférico. Rev Bras Saúde Ocup abr./jun. 1994;22(82):35-44.

8. Castãno JG, Fernandez CC. Alteraciones de salud en trabajadores expuestos a ruidos y vibraciones en ferrocarriles nacionales. Rev Cuba Hig Epidemiol 1989;27(1):87-95.

9. Ferraz NM. A questão da informação na conservação auditiva: a perspectiva da trabalhador portador de perda auditiva induzida pelo ruído. São Paulo, 1995. Tese (Mestrado em Distúrbios da Comunicação), Pontifícia Universidade Católica de São Paulo.
10. Fiorini AC. Conservação auditiva: estudo sobre o monitoramento audiométrico em trabalhadores de uma indústria metalúrgica. São Paulo, 1994. Dissertação (Mestrado em Distúrbios da Comunicação), Pontifícia Universidade Católica de São Paulo.

11. Gerges SNY. Efeitos nocivos - A audição e as conseqüências das vibrações no corpo humano. Proteção 1997;jul:56-62.

12. Hempstock TI, O'Connor DE. Assessment of Hand Transmitted Vibration. Ann Occup Hyg 1978;21:57-67.

13. Hétu R, Phaneuf R. An epidemiological perspective of the causes of hearing loss among industrial workers. J Otolaryngol 1990;19:1.

14. Iki M. Vibration induced white finger as a risk factor for hearing loss and postural instability. Nagoya J Med Sci 1994 (Suppl.) 57:137-145.

15. International Organization For Standardization. Acoustics. Whole body vibration. ISO 2631, Geneve, 1997.

16. International Organization For Standardization. Acoustics. Reference zero for the calibration of audiometric equipament. Part 1: Reference equivalent threshold sound pressure levels for pure tone and supra-aural earphones. ISO R 389 1:1998.

17. International Organization For Standardization. Acoustics. Standard reference zero for the calibration of pure-tone bone conduction audiometers. ISO 7566,1987.

18. Matoba T. Pathophysiology and clinical picture of Hand-Arm vibration Syndrome in Japanese workers. Nagoya J Med Sci 1994 (Suppl.) Japan;57:19-26.

19. Miyakita T, Miura H, Futatsuka M. Noise-induced hearing loss in relation to vibration-induced white finger in chain saw workers. Scand J Work Environ Heath 1997;13:32-6.

20. Morata TC, Lemaster GK. Epidemiologic considerations in the evaluation of occupational hearing loss. Occup Med State Art Rev 1995;10(3):641-56.

21. Murata K, Araki S, Aono H. Central and peripheral nervous system effects of hand-arm vibrating tool operation. A study of brainstem auditory-evoked potencial and peripheral nerve conduction. Int Arch Occup Environ Health 1990;62(3):183-7.

22. National Institute For Occupational Safety And Health - NIOSH. Criteria for a Recommended Standard Occupational Exposure to Hand-Arm Vibration. U. S. Department of Health and Human Services, Cincinnati, Ohio, Sep. 1989.

23. National Institute For Occupational Safety And Health - NIOSH. Preventing Occupational Hearing Loss. A Pratical guide. U. S. Department of Health and Human Services, Cincinnati, Ohio, 1996.

24. National Institute For Occupational Safety And Health - NIOSH. Criteria for a Recommended Standard Occupational Noise Exposure Revised Criterion. U. S. Department of Health and Human Services, Cincinnati, Ohio, 1998.

25. Pekkarinen J. Noise impulse noise, and other physical factors combined effects on hearing. Occup Med: State e Art Reviews 1995;10(2):545-59.

26. Pyykko I, Starch J, Farkkila M, Hoikkala M, Korhoonen O. and Nurminen. Hand arm vibration in the etiology of hearing loss in lumberjacks. Br J Ind Med 1981;38:281-9.

27. Pyykko I, Farkkika M, Inaba R et al. Effect of hand-arm vibration on inner ear and cardiac functions in man. Nagoya J Med Sci 1994 (Suppl.) 57:113-9.

28. Souza MT. Efeitos auditivos provocados pela interação entre ruído e solventes: uma abordagem preventiva em audiologia voltada à saúde do trabalhador. São Paulo, 1994. Dissertação (Mestrado em Distúrbios da Comunicação), Pontifícia Universidade Católica de São Paulo.

29. Starck J, Pekkarinen J, Pyykko I. Impulse noise and hand-arm vibration in relation to sensory neural hearing loss. Scand $\mathrm{J}$ Work Environ Health 19881;4:265-71.

30. Zhu S, Sakakibara H, Yamada S. Combined effects of hand-arm vibration and noise on temporary threshold shifts of hearing in healthy subjects. Int Arch Occup Environ Health 1997 Japan, 6(69):433-6. 